\title{
Explicit soliton-black hole correspondence for static configurations
}

\author{
Shabnam Beheshti*and Floyd L. Williams ${ }^{\dagger}$ \\ Department of Mathematics and Statistics \\ University of Massachusetts \\ Amherst, MA 01003
}

July 17, 2021

\begin{abstract}
We construct an explicit map that transforms static, generalized sine-Gordon metrics to black hole type metrics. This, in particular, provides for a further description of the Cadoni correspondence (which extends the Gegenberg-Kunstatter correspondence) of soliton solutions and extremal black hole solutions in $2 \mathrm{D}$ dilaton gravity.

PACS numbers: 04.60.Kz, 04.70.Bw, 05.45.Yv, 11.10.Kk
\end{abstract}

\section{Introduction}

An interesting, intriguing connection between Euclidean N-soliton sine-Gordon solutions and Lorentzian black hole solutions in Jackiw-Teitelboim dilaton gravity has been established by J. Gegenberg and G. Kunstatter 4, 5, 6. In case $\mathrm{N}=1$, a concrete transformation was constructed that explicates this connection [9]; see also 10, 11]. The construction of such a transformation in general seems to be a difficult problem as it involves, in particular, finding explicit solutions of a system of dilaton field equations. Recently, these field equations were solved for a kink-antikink soliton (similar solutions were found in [5]), and thus an explicit transformation was also constructed in this case [2] that further implements the work in [4] [5].

We present a transformation $\Psi$ that takes any generalized static sine-Gordon type metric to a black hole type metric. In particular we present additional solutions of the dilaton field equations (even in the non-static case) and a further description of the M. Cadoni correspondence [3] between extremal black

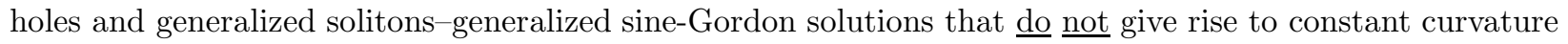
space-times, as in the Gegenberg-Kunstatter discussion.

We dedicate this paper to the memory of Professor Melvyn Berger-friend, and outstanding scholar in non-linear phenomenon.

\section{Field equations for 2D dilaton gravity}

Given a potential function $V(r)$ and $l>0$, we consider the general two-dimensional dilaton gravity theory with action integral

$$
I(\tau, g)=\frac{1}{2 G} \int d^{2} x \sqrt{-g}\left(\tau R(g)+\frac{V \circ \tau}{l^{2}}\right),
$$

\footnotetext{
*beheshti@math.umass.edu

†williams@math.umass.edu
} 
for which the equations of motion are

$$
\begin{aligned}
R(g)+\frac{V^{\prime} \circ \tau}{l^{2}} & =0 \\
\nabla_{\mu} \nabla_{\nu} \tau+\frac{1}{2 l^{2}} g_{\mu \nu}(V \circ \tau) & =0
\end{aligned}
$$

for the dilaton field $\tau(T, r)$ and metric $g$ with scalar curvature $R=R(g)$. For $\tau(T, r)=\frac{r}{l}$, for example, and a constant $C$ one has the well-known solution [1, 7 ]

$$
d s^{2}=-\left[-J\left(\frac{r}{l}\right)-C\right] d T^{2}+\left[-J\left(\frac{r}{l}\right)-C\right]^{-1} d r^{2}
$$

for $J^{\prime}(r)=V(r)$. Actually, $C=-l^{2}|\nabla \tau|^{2}-J \circ \tau$, and $\frac{C}{2 l}$ can be interpreted as the energy of the solution. For spherically symmetric gravity, for example, with $V(r)=-\frac{\gamma}{\sqrt{r}}, \gamma>0$, one takes $l=$ the Planck length $l_{P}$.

For $g$ given by

$$
d s^{2}=\cos ^{2} \frac{u(x, t)}{2} d x^{2}-\sin ^{2} \frac{u(x, t)}{2} d t^{2}
$$

and for $\Delta=\frac{\partial^{2}}{\partial x^{2}}+\frac{\partial^{2}}{\partial t^{2}}$,

$$
R=\frac{2 \Delta u}{\sin u}
$$

by our sign convention for the scalar curvature, which is opposite the sign of $R$ in the references [3, 5], and the equations in (3) are

$$
\begin{aligned}
\tau_{x t}+\frac{1}{2} \tan \left(\frac{u}{2}\right) u_{t} \tau_{x}-\frac{1}{2} \cot \left(\frac{u}{2}\right) u_{x} \tau_{t} & =0 \\
\tau_{x x}+\frac{1}{2} \tan \left(\frac{u}{2}\right) u_{x} \tau_{x}+\frac{1}{2} \cot \left(\frac{u}{2}\right) u_{t} \tau_{t}+\frac{1}{2 l^{2}} \cos ^{2}\left(\frac{u}{2}\right)(V \circ \tau) & =0 \\
\tau_{t t}-\frac{1}{2} \tan \left(\frac{u}{2}\right) u_{x} \tau_{x}-\frac{1}{2} \cot \left(\frac{u}{2}\right) u_{t} \tau_{t}-\frac{1}{2 l^{2}} \sin ^{2}\left(\frac{u}{2}\right)(V \circ \tau) & =0 .
\end{aligned}
$$

By equations (2), (6) and by addition of equations (8), (9) on obtains

$$
\begin{aligned}
\Delta u & =-\frac{1}{2 l^{2}}\left(V^{\prime} \circ \tau\right) \sin u \\
\Delta \tau & =-\frac{1}{2 l^{2}}(V \circ \tau) \cos u,
\end{aligned}
$$

equation (10) being a generalized sine-Gordon equation. Following M. Cadoni [3], we shall be interested in static field solutions $u(x, t)=u(x), \tau(x, t)=\tau(x)$ in which case (10), (11) reduce to the system

$$
\begin{aligned}
u^{\prime \prime}(x) & =-\frac{1}{2 l^{2}} V^{\prime}(\tau(x)) \sin u(x) \\
\tau^{\prime \prime}(x) & =-\frac{1}{2 l^{2}} V(\tau(x)) \cos u(x),
\end{aligned}
$$

which has first integrals

$$
\begin{aligned}
u^{\prime}(x) & =-\frac{A}{l} V(\tau(x)) \\
\tau^{\prime}(x) & =\frac{1}{2 l A} \sin u(x),
\end{aligned}
$$

for any constant $A \neq 0$. Note that by (14), (15) one easily deduces that

$$
\left.\frac{d}{d x}\left[A^{2} J(\tau(x))\right)+\sin ^{2} \frac{u(x)}{2}\right]=0
$$


for $J^{\prime}(x)=V(x)$, which gives the conservation law

$$
A^{2} J(\tau(x))+\sin ^{2} \frac{u(x)}{2}=\text { a constant }
$$

which we express as

$$
\sin ^{2} \frac{u(x)}{2}=-A^{2}[J(\tau(x))+C]
$$

for a constant C. Using equations (15), (18) one can also deduce that

$$
\tau^{\prime}(x)^{2}=-\frac{1}{l^{2}}[J(\tau(x))+C]\left\{1+A^{2}[J(\tau(x))+C]\right\} .
$$

Equations (18), (19), which we have deduced by a conservation law, compare with equations (16), (17) in [3] where $-K,-V$, and $\Phi$ there are our $J, V$, and $\tau$, respectively.

\section{The Transformations $\Psi$}

In the static case under consideration we write the metric in (5) as

$$
d s_{\text {sol }}^{2}=\cos ^{2} \frac{u(x)}{2} d x^{2}-\sin ^{2} \frac{u(x)}{2} d t^{2},
$$

where the subscript "sol" suggests the word soliton -given equation (10). We look for an explicit map $\Psi=\left(\psi_{1}, \psi_{2}\right)$ and its inverse $\Theta=\left(\theta_{1}, \theta_{2}\right)$ such that under the change of variables $x=\psi_{1}(T, r), t=\psi_{2}(T, r)$, the metric $d s_{\text {sol }}^{2}$ in (20) is transformed to the metric

$$
d s_{b h}^{2}=-\left[-J\left(\frac{r}{l}\right)-C\right] d T^{2}+\left[-J\left(\frac{r}{l}\right)-C\right]^{-1} d r^{2}
$$

in (4) for the $\mathrm{C}$ in equations (18), (19), where the subscript "bh" suggests now some kind of generic extremal black hole. Conversely, under the change of variables $T=\theta_{1}(x, t), r=\theta_{2}(x, t), d s_{b h}^{2} \longrightarrow d s_{s o l}^{2}$. It turns out that, in contrast to the more difficult non-static case, $\Psi$ and $\Theta$ can be chosen to assume the following somewhat simple form:

$$
\begin{aligned}
\psi_{1}(T, r) & =\tau^{-1}\left(\frac{r}{l}\right), & \psi_{2}(T, r) & =\frac{T-\theta_{0}}{A} \\
\theta_{1}(x, t) & =A t+\theta_{0}, & \theta_{2}(x, t) & =l \tau(x)
\end{aligned}
$$

for $u(x)$ in (20) that satisfies equation (14) and $\tau(x)$ that satisfies equation (15); hence $u(x)$ and $\tau(x)$ will solve the system (12) and (13). $\tau^{-1}$ is the inverse function of $\tau$ and $\theta_{0}$ is any constant. The verification that the transformations in (22), (23) indeed do work relies heavily on the equations (18), (19), which as we have seen are implied by equations (14), (15).

As a simple, but important example, choose $V(x)=-2 x$ (which provides for the Jackiw-Teitelboim model), and choose

$$
u(x)= \pm 4 \arctan e^{\frac{x-x_{0}}{l}}, \quad \tau(x)= \pm \operatorname{sech}\left(\frac{x-x_{0}}{l}\right)
$$

which solve equations (14), (15) for $A=1$ (and which therefore solve the sine-Gordon equation $u^{\prime \prime}(x)=$ $\frac{1}{l^{2}} \sin u(x)$ (12) and equation (13)). Equation (18) holds if and only if $C=0$. By equation (22), the transformation of variables

$$
\begin{aligned}
& x=\psi_{1}(T, r)=x_{0}+l \log \left[\frac{l+\sqrt{l^{2}-r^{2}}}{ \pm r}\right] \\
& t=\psi_{2}(T, r)=T
\end{aligned}
$$


(where we choose $\theta_{0}=0$ ) takes the soliton metric $d s_{\text {sol }}^{2}$ in (20) (for $u(x)$ given in (24)) to the extremal black hole metric $d s_{b h}^{2}=-\frac{r^{2}}{l^{2}} d T^{2}+\frac{l^{2}}{r^{2}} d r^{2}$; here $J(x)=-x^{2}$. Similarly by (23) under the transformation of variables $T=\theta_{1}(x, t)=t, r=\theta_{2}(x, t)= \pm l \operatorname{sech}\left(\frac{x-x_{0}}{l}\right), d s_{b h}^{2} \longrightarrow d s_{\text {sol }}^{2}$. This transformation and its inverse $\Psi$ in (25), (26) can be seen as implementing the Cadoni correspondence for the present example. Note a minor typing error in equation (31) of [3]: there one should have $\Phi^{-1}= \pm \cosh \left(\lambda\left(x-x_{0}\right)\right.$ ) (instead of $\Phi^{-1}=\cosh \left(\lambda\left(x-x_{0}\right)\right)$, as the minus sign is needed for the minus in (24)).

As pointed out in [3], a complete correspondence between 2D space-time structures of the dilaton gravity theory and solutions in generalized sine-Gordon field theory requires a consideration of the metric (5) and of the metric

$$
d s^{2}=\cosh ^{2} \frac{u(x, t)}{2} d x^{2}-\sinh ^{2} \frac{u(x, t)}{2} d t^{2}
$$

as well, for the sinh-Gordon model. Here for $\square=\frac{\partial^{2}}{\partial x^{2}}-\frac{\partial^{2}}{\partial t^{2}}, R=\frac{2 \square u}{\sinh u}$ and the equations (2), (3) are now

$$
\begin{aligned}
\square u+\frac{1}{2 l^{2}}\left(V^{\prime} \circ \tau\right) \sinh u & =0 \\
\tau_{x t}-\frac{1}{2} \tanh \left(\frac{u}{2}\right) u_{t} \tau_{x}-\frac{1}{2} \operatorname{coth}\left(\frac{u}{2}\right) u_{x} \tau_{t} & =0 \\
\tau_{x x}-\frac{1}{2} \tanh \left(\frac{u}{2}\right) u_{x} \tau_{x}-\frac{1}{2} \operatorname{coth}\left(\frac{u}{2}\right) u_{t} \tau_{t}+\frac{1}{2 l^{2}} \cosh ^{2}\left(\frac{u}{2}\right)(V \circ \tau) & =0 \\
\tau_{t t}-\frac{1}{2} \tanh \left(\frac{u}{2}\right) u_{x} \tau_{x}-\frac{1}{2} \operatorname{coth}\left(\frac{u}{2}\right) u_{t} \tau_{t}-\frac{1}{2 l^{2}} \sinh ^{2}\left(\frac{u}{2}\right)(V \circ \tau) & =0,
\end{aligned}
$$

from which one obtains in the static case the system

$$
\begin{aligned}
u^{\prime \prime}(x) & =-\frac{1}{2 l^{2}} V^{\prime}(\tau(x)) \sinh u(x) \\
\tau^{\prime \prime}(x) & =-\frac{1}{2 l^{2}} V(\tau(x)) \cosh u(x)
\end{aligned}
$$

with first integrals

$$
\begin{aligned}
u^{\prime}(x) & =-\frac{A}{l} V(\tau(x)) \\
\tau^{\prime}(x) & =\frac{1}{2 A l} \sinh u(x),
\end{aligned}
$$

that compare with equations (14), (15). Equations (18), (19) are replaced by

$$
\begin{aligned}
\sinh ^{2} \frac{u(x)}{2} & =-A^{2}[J(\tau(x))+C] \\
\tau^{\prime}(x)^{2} & =-\frac{1}{l^{2}}[J(\tau(x))+C]\left\{1-A^{2}[J(\tau(x))+C]\right\}
\end{aligned}
$$

for a suitable constant $C$. For this $C$, and for $u(x), \tau(x)$, that solve (34), (35) (hence $u(x), \tau(x)$ also solve equations (32), (33) ) one can check that for the metric $d s^{2}$ in (27), $d s^{2} \longrightarrow d s_{b h}^{2}$ in (21), under the change of variables $(x, t) \longrightarrow \Psi(T, r)=\left(\psi_{1}(T, r), \psi_{2}(T, r)\right)$, where

$$
\begin{aligned}
\psi_{1}(T, r) & =\tau^{-1}\left(\frac{r}{l}\right), & \psi_{2}(T, r) & =\frac{T-\theta_{0}}{A} \\
\theta_{1}(x, t) & =A t+\theta_{0}, & \theta_{2}(x, t) & =l \tau(x) ;
\end{aligned}
$$

here $\Theta=\left(\theta_{1}, \theta_{2}\right)=\Psi^{-1}$. Thus $\Psi, \Theta$ here have the same form as the $\Psi, \Theta$ in (22), (23). 


\section{The Sinh- $\Phi$ model and other examples}

Another model is defined by the potential $V(x)=-\sinh (2 x)$. Equations (14), (15) are solved by

$$
\begin{aligned}
& u(x)=\pi+2 \arctan \left[\sqrt{2} \sinh \left(\frac{x-x_{0}}{l}\right)\right] \\
& \tau(x)=\operatorname{arctanh}\left[\frac{1}{\sqrt{2}} \operatorname{sech}\left(\frac{x-x_{0}}{l}\right)\right]
\end{aligned}
$$

for $A=1$, and equation (18) holds for $C=\frac{1}{2} . J(x)=-\frac{1}{2} \cosh (2 x)$. The extremal black hole solution $d s_{b h}^{2}$ corresponding to the solutions (40), (41), in the Cadoni correspondence, is given by equation (21):

$$
\begin{aligned}
d s_{b h}^{2} & =-\left[\frac{1}{2} \cosh \left(\frac{2 r}{l}\right)-\frac{1}{2}\right] d T^{2}+\left[\frac{1}{2} \cosh \left(\frac{2 r}{l}\right)-\frac{1}{2}\right]^{-1} d r^{2} \\
& =-\sinh ^{2}\left(\frac{r}{l}\right) d T^{2}+\sinh ^{-2}\left(\frac{r}{l}\right) d r^{2}
\end{aligned}
$$

as in equation (40) of [3]. A change of variables $x=\psi_{1}(T, r), t=\psi_{2}(T, r)$ that takes $d s_{\text {sol }}^{2}$ in (20) (for $u(x)$ in (40)) directly to $d s_{b h}^{2}$ in (42) is given by equation (22), where we note that $\tau^{-1}(x)=x_{0}+l \operatorname{arcsech}(\sqrt{2} \tanh (x))$ for $\tau(x)$ in (41):

$$
\begin{aligned}
& \psi_{1}(T, r)=x_{0}+l \log \left[\frac{1+\sqrt{1-2 \tanh ^{2} \frac{r}{l}}}{\sqrt{2} \tanh \frac{r}{l}}\right] \\
& \psi_{2}(T, r)=T,
\end{aligned}
$$

where again we take $\theta_{0}=0$.

Going back to the Jackiw-Teitelboim model with $V(x)=-2 x$, one can obtain another solution $u(x)$, $\tau(x)$ of the field equations (12), (13) - one of independent interest that involves the Jacobi elliptic funcitons sn, cn, and dn [8]. For this, given $A \neq 0$ (as in equations (14), (15)) and a constant $E>0$, define

$$
\begin{aligned}
B & =\frac{A^{2} E}{4 l^{2}} \\
K & =1+\frac{2}{A^{2} E} \\
\alpha & =\sqrt{2 K^{2}+2 K \sqrt{K^{2}-1}-1} \\
g(x) & =\left(\sqrt{B} \sqrt{\sqrt{K^{2}-1}-K}\right) x \\
f(x) & =\frac{\operatorname{sn}(g(x), \alpha)}{\sqrt{\sqrt{K^{2}-1}-K}} .
\end{aligned}
$$

Then one can show that the pair

$$
\begin{aligned}
u(x) & =4 \arctan f(x) \\
\tau(x) & =\frac{\sqrt{E} \operatorname{cn}(g(x), \alpha) \operatorname{dn}(g(x), \alpha)}{1+\frac{\operatorname{sn}^{2}(g(x), \alpha)}{\sqrt{K^{2}-1}-K}}
\end{aligned}
$$

solves equations (14), (15). Since $\operatorname{sn}(0, \alpha)=0$ and $\operatorname{cn}(0, \alpha)=\operatorname{dn}(0, \alpha)=1$, we see that $u(0)=0$ and $\tau(0)=\sqrt{E}$. Also, $J(x)=-x^{2}$, which means that in equation (18) we can conclude that $C=\tau(0)^{2}=E$, and that the solution in (21) corresponding to (46), (47) is given by

$$
d s_{b h}^{2}=-\left[\frac{r^{2}}{l^{2}}-E\right] d T^{2}+\left[\frac{r^{2}}{l^{2}}-E\right]^{-1} d r^{2},
$$


which is a black hole with positive mass $E$. In this case it is easier to compute the inverse tranformation $\Theta=\Psi^{-1}$ in (23) which is simply $l \tau$ for $\tau$ in (47).

The string-inspired gravity model, with $V(x)=-\gamma, \gamma>0$, gives rise to the (non-soliton) example

$$
u(x)=\frac{A \gamma}{l} x+b, \quad \tau(x)=-\frac{1}{2 A^{2} \gamma} \cos \left(\frac{A \gamma}{l} x+b\right)+c
$$

that solves $\left(14,(15) . J(x)=-\gamma x\right.$ and the choice of $x=-\frac{b l}{A \gamma}$ in (18) gives $C=-\frac{1}{2 A^{2}}+\gamma c$, by which one obtains the solution (see (21))

$$
d s_{b h}^{2}=-\left[\frac{\gamma}{l} r+\frac{1}{2 A^{2}}-\gamma c\right] d T^{2}+\left[\frac{\gamma}{l} r+\frac{1}{2 A^{2}}-\gamma c\right]^{-1} d r^{2}
$$

$\Psi(T, r)$ in (22) assumes the form

$$
\begin{aligned}
\psi_{1}(T, r) & =\frac{l}{A \gamma}\left\{-b+\arccos \left[2 A^{2} \gamma\left(c-\frac{r}{l}\right)\right]\right\} \\
\psi_{2}(T, r) & =\frac{T-\theta_{0}}{A} .
\end{aligned}
$$

\section{Remarks on non-static solutions}

Non-static solutions of the field equations (7), (8), (9) (for string-inspired, spherically symmetric, and JackiwTeitelboim (JT) gravity) are given in 2], that complement 2-soliton solutions found in 5 for JT gravity. For example, given $m$ and $v>0$ (which we regard as mass and velocity parameters) set $a^{2}=1+v^{2}$ and define

$$
\begin{aligned}
u(x, t) & =4 \arctan \left[\frac{v \sinh (a m x)}{a \cos (v m t)}\right] \\
\tau(x, t) & =\frac{4 v^{2} a m[\sin (v m t)] \sinh (a m x)}{a^{2} \cos ^{2}(v m t)+v^{2} \sinh ^{2}(a m x)} .
\end{aligned}
$$

This pair solves the system (7)-(9) for $V(x)=-x, l=\frac{1}{\sqrt{2} m}$, as shown in [2, where a transformation $\Psi$ is also constructed that takes the metric in (5) to that in (4), for an appropriate value of $C$. Another application for such transformations $\Psi$ is toward the construction of exact solutions of field equations defined by the Laplacian $\square_{\text {sol }}^{+}$of the metric (5). In the static case at hand, for example, one can prove the following commutivity: let $\square_{\text {sol }}^{-}$denote the Laplacian of the metric in equation (27), let $\square_{b h}$ denote the Laplacian of the metric in equation (4), and let $D_{\tau}^{ \pm}$denote the domains

$$
D_{\tau}^{ \pm}=\left\{(x, t) \in \mathbb{R}^{2} \mid \pm \tau^{\prime}(x)>0\right\} .
$$

Then for a function $f(T, r)$ and the transformation $\Theta$ in (23) (which we have observed is the same as that in (39)), one has

$$
\begin{aligned}
& \square_{\text {sol }}^{+}(f \circ \Theta)=\left(\square_{b h} f\right) \circ \Theta \text { on } D_{\tau}^{+} \\
& \square_{\text {sol }}^{-}(f \circ \Theta)=\left(\square_{b h} f\right) \circ \Theta \text { on } D_{\tau}^{-} .
\end{aligned}
$$

This is based on the somewhat remarkable property that these $\Theta=\Psi^{-1}$, in principle, commute with $\square_{\text {sol }}$ and $\square_{b h}$, which means that they are also transformations of solution spaces. Details of this commutation property are found in [11, in the JT case, where some solutions of the equation $\square_{\text {sol }} \phi=\mu \phi$ are also presented. 


\section{References}

[1] T. Banks and M. O'Laughlin, Nucl. Phys. B362, 649 (1991).

[2] S. Beheshti, Ph.D. Thesis, University of Massachusetts, Amherst, MA (2006).

[3] M. Cadoni, Phys. Rev. D58 104001, (1998).

[4] J. Gegenberg and G. Kunstatter, Phys. Lett. B413, 274 (1997).

[5] J. Gegenberg and G. Kunstatter, Phys. Rev. D58 124010, 274 (1998).

[6] J. Gegenberg and G. Kunstatter, from Solitons: Properties, Dynamics, Interactions, Applications, M. Mackenzie, R. Paranjape, and W. Zakrzewski, Editors. Chapter 14, 99 (2000).

[7] D. Louis-Martinez, J. Gegenberg, and G. Kunstatter, Phys. Lett. B321, 193 (1994).

[8] H. Hancock, Lectures on the Theory of Elliptic Functions, Dover Pub. (1958).

[9] F. Williams, from Quantum Field Theory Under the Influence of External Conditions, K. Milton, Editor, 370, Rinton Press (2004).

[10] F. Williams, from Proceedings of the Fourth International Conference on Mathematical Physics, Rio de Janeiro, Brazil (2004), Proceedings of Science, http://pos.sissa.it.

[11] F. Williams, from Trends in Soliton Research, L. Chen, Editor, Chapter 1, 1 Nova Science Pub. (2006). 